\title{
Effects of balance training on balance performance in youth: role of training difficulty
}

\author{
Simon Schedler ${ }^{*}$ (D), Florian Tenelsen, Laura Wich and Thomas Muehlbauer
}

\begin{abstract}
Background: Cross-sectional studies have shown that balance performance can be challenged by the level of task difficulty (e.g., varying stance conditions, sensory manipulations). However, it remains unclear whether the application of different levels of task difficulty during balance training (BT) leads to altered adaptations in balance performance. Thus, we examined the effects of BT conducted under a high versus a low level of task difficulty on balance performance.
\end{abstract}

Methods: Forty male adolescents were randomly assigned to a BT program using a low (BT-low: $n=20$; age: $12.4 \pm$ $2.0 \mathrm{yrs}$ ) or a high (BT-high: $n=20$; age: $12.5 \pm 2.5 \mathrm{yrs}$ ) level of balance task difficulty. Both groups trained for 7 weeks (2 sessions/week, 30-35 min each). Pre- and post-training assessments included measures of static (one-legged stance [OLS] time), dynamic (10-m gait velocity), and proactive (Y-Balance Test [YBT] reach distance, Functional Reach Test [FRT]; Timed-Up-and-Go Test [TUG]) balance.

Results: Significant main effects of Test (i.e., pre- to post-test improvements) were observed for all but one balance measure (i.e., 10-m gait velocity). Additionally, a Test $x$ Group interaction was detected for the FRT in favor of the BT-high group $(\Delta+8 \%, p<0.001, d=0.35)$. Further, tendencies toward significant Test $\times$ Group interactions were found for the YBT anterior reach (in favor of BT-high: $\Delta+9 \%, p<0.001, d=0.60$ ) and for the OLS with eyes opened and on firm surface (in favor of BT-low: $\Delta+31 \%, p=0.003, d=0.67$ ).

Conclusions: Following 7 weeks of BT, enhancements in measures of static, dynamic, and proactive balance were observed in the BT-high and BT-low groups. However, BT-high appears to be more effective for increasing measures of proactive balance, whereas BT-low seems to be more effective for improving proxies of static balance.

Trial registration: Current Controlled Trials ISRCTN83638708 (Retrospectively registered 19th June, 2020).

Keywords: Postural control, Adolescence, Intervention, Dose-response relationship

\section{Background}

The effectiveness of balance training (BT) for improving different components of balance performance in children and adolescents has been shown by several original studies [1-4] and these findings have been summarized in systematic reviews $[5,6]$. Contrary, recommendations on

* Correspondence: simon.schedler@uni-due.de

Division of Movement and Training Sciences/Biomechanics of Sport, University of Duisburg-Essen, Gladbecker Str. 182, 45141 Essen, Germany how to design BT with respect to different load dimensions (e.g., training volume, training intensity) in order to be most effective in children and adolescents are rather unspecific and have only been derived from review articles [5, 7]. For example, a reduction in the base of support / sensory input and the inclusion of unstable surfaces / cognitive and motor interference tasks have been proposed as being effective means to increase task difficulty and thus enhance balance performance in

(c) The Author(s). 2020 Open Access This article is licensed under a Creative Commons Attribution 4.0 International License, which permits use, sharing, adaptation, distribution and reproduction in any medium or format, as long as you give appropriate credit to the original author(s) and the source, provide a link to the Creative Commons licence, and indicate if changes were made. The images or other third party material in this article are included in the article's Creative Commons licence, unless indicated otherwise in a credit line to the material. If material is not included in the article's Creative Commons licence and your intended use is not permitted by statutory regulation or exceeds the permitted use, you will need to obtain permission directly from the copyright holder. To view a copy of this licence, visit http://creativecommons.org/licenses/by/4.0/ The Creative Commons Public Domain Dedication waiver (http://creativecommons.org/publicdomain/zero/1.0/) applies to the data made available in this article, unless otherwise stated in a credit line to the data. 
youth [7]. However, although these recommendations seem to be justified based on the available literature, empirical evidence is still lacking. Indeed, Gebel et al. [5] conducted a systematic review with meta-analysis analyzing the effects and dose-response relationships of BT on balance performance in youth. Yet, using this approach dose-response relationships are compared indirectly instead of directly. In other words, results from a study with a short intervention period were for instance compared to those from a study with a long one, but there was no comparison of the effects of different intervention periods within a single study. Additionally, doseresponse relationships of BT in youth could only be quantified for certain training modalities (i.e., training period, training frequency). To summarize, there is a need for research which (i) directly compares different training modalities within a single study and (ii) investigates load dimensions that have not been analyzed so far.

One of these overlooked load dimensions is "training difficulty". This is especially surprising as changing the level of balance task demand (e.g., varying stance conditions or manipulating the sensory input) has been described as being one way to design varied and challenging BT $[7,8]$. Further, cross-sectional studies [9-13] showed that balance performance can be challenged by the level of task difficulty. In this regard, Muehlbauer et al. [10] reported an increase in postural sway when the base of support was reduced (i.e., from bipedal stance over step and tandem stance to unipedal stance) and the sensory input was altered (i.e., from standing with eyes opened on firm ground over eyes opened on foam ground to eyes closed on firm ground). However, the effects of conducting BT with different balance task demands on balance performance in youth have not been investigated in the context of regular BT lasting several weeks.

Thus, the aim of the present study was to investigate the effects of BT conducted under a high versus a low level of task difficulty on balance performance in youth. We expected that both exercise conditions will result in enhanced balance performance but the improvement will be larger in the group that used a high compared to a low level of balance task difficulty during training.

\section{Methods}

\section{Participants}

Using G*Power [14], the power analysis $(f=0.25, \alpha=$ $0.05,1-\beta=0.80$, number of groups: $n=2$, number of measurements: $n=2$, correlation between testing: $r=$ 0.50 , drop-out rate per group: $10 \%$ due to injury reasons not attributable to treatments) revealed that a total sample size of $N=37$ participants (i.e., $n=18-19$ per group) would be sufficient to detect statistically significant training effects. Therefore, forty healthy male adolescents from a local sports club were assessed for eligibility and randomly assigned to either a BT-low $(n=20$; age: $12.4 \pm 2.0$ years, body mass: $61.0 \pm 22.6 \mathrm{~kg}$, body height: $159.0 \pm 13.7 \mathrm{~cm}$; body mass index: $\left.23.9 \pm 7.7 \mathrm{~kg} / \mathrm{m}^{2}\right)$ or a BT-high ( $n=20$; age: $12.5 \pm 2.5$ years, body mass: $64.5 \pm$ $22.1 \mathrm{~kg}$, body height: $162.4 \pm 14.2 \mathrm{~cm}$; body mass index: $24.0 \pm 6.5 \mathrm{~kg} / \mathrm{m}^{2}$ ) group using Research Randomizer (www.randomizer.org) (Fig. 1). Participants were free of any known neurological, musculoskeletal, or orthopedic diseases. Over the course of the study, a participant of the BT-high group dropped out due to an injury not related to the intervention. In addition, one subject of the BT-low group performed only selected items during post-testing and was thus excluded from statistical analyses. The study was conducted in accordance with the CONSORT guidelines [15]. Participant recruitment began in January 2019 and the assessments and intervention were carried out the following months. Participants' assent and parents' written informed consent were obtained prior to the start of the study. The $\mathrm{Hu}$ man Ethics Committee at the University of DuisburgEssen, Faculty of Educational Sciences approved the study protocol (approval code: TM_06_12_2018).

\section{Testing procedures}

The pre- and post-testing was conducted in a gym hall by the same skilled assessors (graduated sport scientists) before and after the 7-weeks training period (Fig. 2a). All participants received standardized verbal instructions and a visual demonstration regarding the testing procedure that included assessment of anthropometric variables, static, dynamic and proactive balance (Fig. 2b). One practice followed by one data-registration trial was performed for each test, unless otherwise stated. All subject conducted a standardized 10-min warm-up prior to each testing that consisted of submaximal running (e.g., skipping, hip in/out) and balance exercises (e.g., forward/backward beam walking, single leg stance on unstable devices).

\section{Assessment of static balance}

For the assessment of static balance, the participants were asked to stand without shoes on their nondominant leg (determined per self-report), hands placed on hips and gaze fixed on a cross on the nearby wall. The participants were instructed to perform the onelegged stance as long as possible but for a maximum of $60 \mathrm{~s}$. The maximal stance time (s) was used for further analysis. Static balance performance was assessed under three different conditions: (1) standing with eyes opened on firm ground; (2) standing with eyes closed on firm ground; (3) standing with eyes opened on foam ground (i.e., AIREX Balance-pad). 


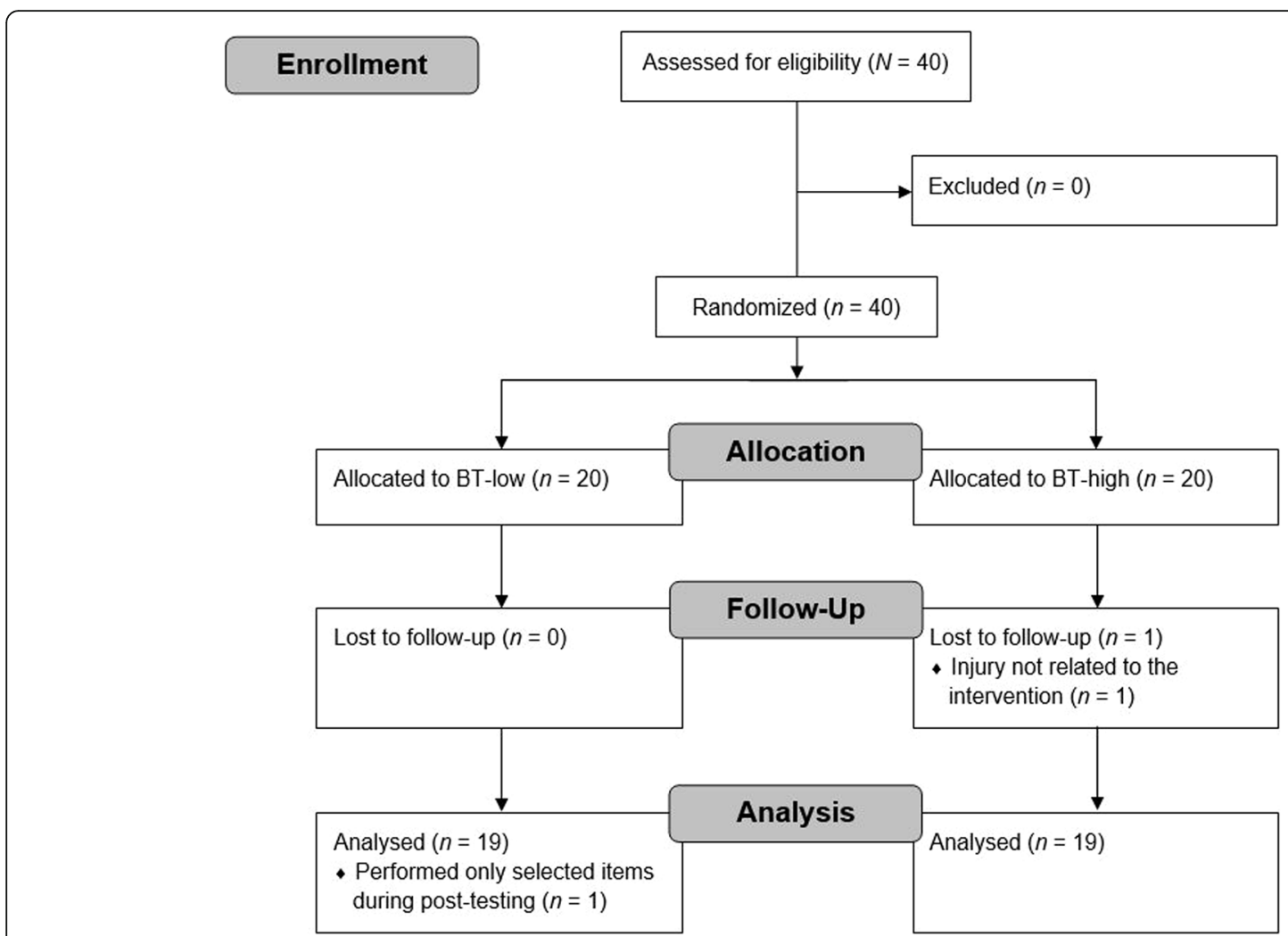

Fig. 1 Flow diagram of the progress through the phases of the study according to the CONSORT statements. BT-low = balance training using a low level of task difficulty; BT-high = balance training using a high level of task difficulty

\section{Assessment of dynamic balance}

Dynamic balance was assessed using the 10-m walk test. The participants were instructed to walk with their preferred speed, initiating and terminating the walk a minimum of one meter before and after the 10-m walkway. The time needed to perform the test was measured with a stopwatch to the nearest $0.01 \mathrm{~s}$. Gait speed $(\mathrm{m} / \mathrm{s})$ was calculated as the 10-m walking distance $(\mathrm{m})$ divided by walking time (s) and used for further analysis.

\section{Assessment of proactive balance}

Proactive balance was assessed by means of the YBalance Test (YBT) Kit (Functional Movement Systems ${ }^{\oplus}$, Chatham, USA). The test kit consists of a centralized stance platform to which three pipes were attached that represent the anterior (AT), posteromedial (PM), and posterolateral $(\mathrm{PL})$ reach directions. Each pipe is marked in $1.0-\mathrm{cm}$ increments for measurement purposes and equipped with a moveable reach indicator. Before the YBT began, the respective lengths of the participants' non-dominant leg was determined in supine position by measuring the distance from the anterior superior iliac spine to the most distal aspect of the medial malleolus [16]. Afterwards, participants were asked to reach with the dominant leg as far as possible in the AT, PM, and
PL directions while standing with their non-dominant leg on the centralized stance platform. A total of six trials (three practice trials followed by three data-collection trials) were executed. The maximal reach distance $(\mathrm{cm})$ per reach direction was used for further analysis In this regard, the normalized maximal reach distance (\% leg length [LL]) per reach direction was calculated by dividing the absolute maximal reach distance $(\mathrm{cm})$ by LL (cm) and then multiplying by 100 . In addition, the normalized (\% LL) composite score (CS) was computed as the sum of the absolute maximal reach distance $(\mathrm{cm})$ per reach direction divided by three times LL $(\mathrm{cm})$ and then multiplied by 100 and used for analysis as well.

Proactive balance was further assessed using the Functional Reach Test (FRT) [17]. The participants' task was to reach with the non-dominant arm (determined by self-report) forward while maintaining a fixed base of support in the standing position. Maximal reach distance (cm) was determined with a measuring tape that was fixated on a wall. Two trials with a 60-s break between trials were performed and the best trial (i.e., largest distance) was used for further analysis.

The Timed-Up-and-Go Test (TUG) was additionally used for the assessment of proactive balance performance [18]. In this regard, the participants were asked to 


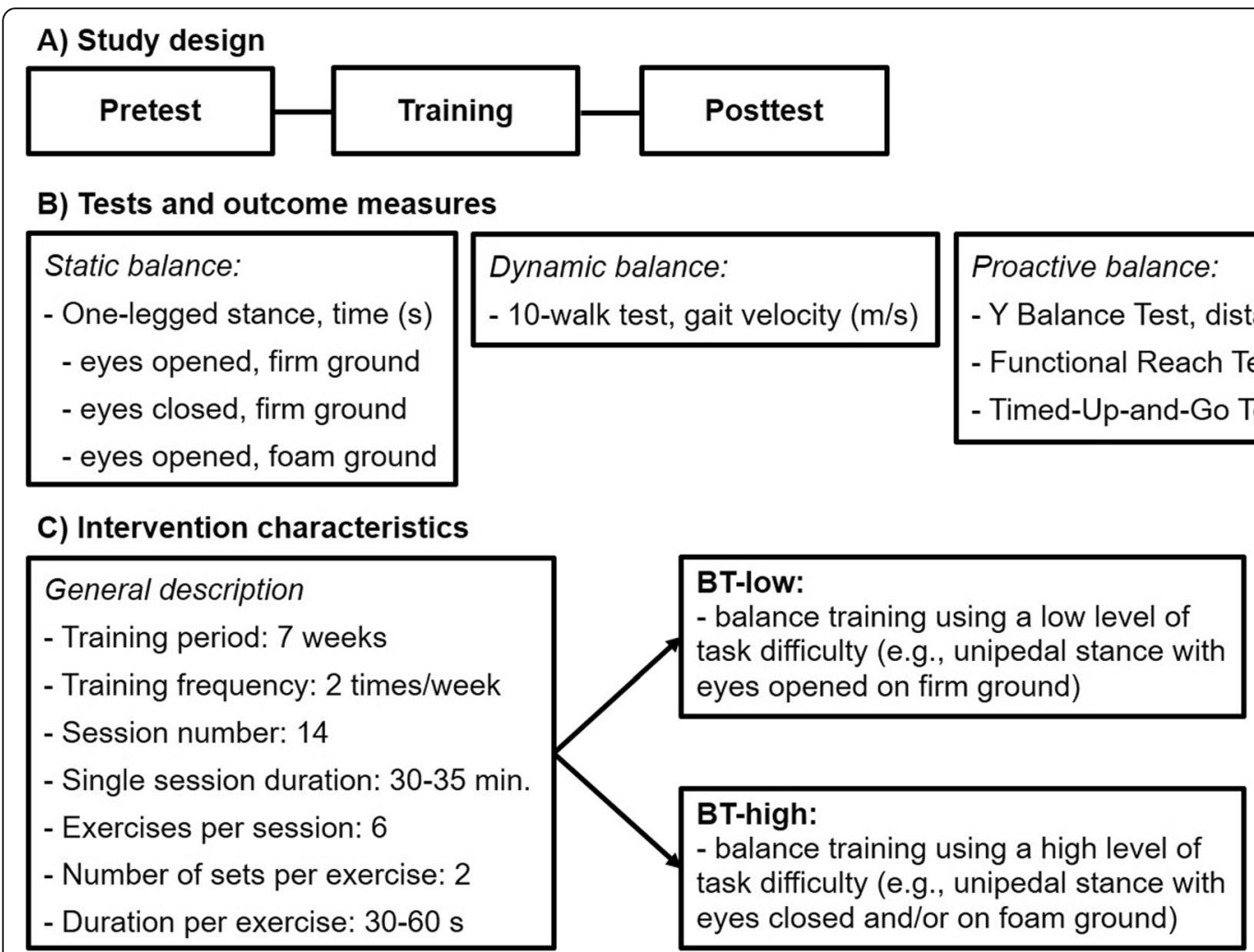

Fig. 2 Schematic description of the study design (a), tests and outcome measures (b), and the intervention characteristics (c). BT-low = balance training using a low level of task difficulty; BT-high = balance training using a high level of task difficulty

rise from a chair, walk three meters, turn around, walk back to the chair and sit down. The time (s) needed to perform the TUG was recorded with a stopwatch to the nearest $0.01 \mathrm{~s}$. Each participant performed two trials with $60 \mathrm{~s}$ in between and the best trial (i.e., shortest time) was used for further analysis.

\section{Balance training programs}

Both groups conducted 7 weeks of BT (2 sessions/week, 30-35 min each) that included six balance exercises per training session (Fig. 2c). After a 15-min warm-up program including general (e.g., skipping, running with hip in/out) and specific (e.g., two-/one-legged stance on unstable devices, forward/backward beam walking) exercises, two sets per balance exercise were performed for $30 \mathrm{~s}$ each with a $60 \mathrm{~s}$ rest period between sets and a $90 \mathrm{~s}$ break between exercises. While participants of the BTlow group performed all balance exercises with a low level of task difficulty, subjects in the BT-high group executed the same or similar exercises using a high level of task difficulty (Table 1). Yet, both groups executed the same training volume (i.e., number of exercises, number of sets per exercise, and duration per set of exercise). Progression during training was achieved by means of increasing exercise duration (i.e., from $30 \mathrm{~s}$ over $45 \mathrm{~s}$ to 60 s), change of stance (i.e., two-legged stance, tandem stance, one-legged stance) and walking (i.e., forward, backward) condition, manipulation of visual input (e.g., eyes opened vs. closed), and concurrent execution of cognitive (i.e., backward counting) or motor (i.e., throwing/catching a ball) tasks. All training sessions ended with a 15-min cool-down that included flexibility exercises and jogging at light intensity.

\section{Statistical analyses}

Descriptive data are reported as group mean values and standard deviations. An univariate analysis of variance (ANOVA) was conducted to test for significant differences in pre-testing values between the two groups. Thereafter, a 2 (Test: pre, post) $\times 2$ (Group: BT-high, BT-low) ANOVA with repeated measures on Test was used. In the case of a significant $(p<0.05)$ or a tendency toward a significant $(.05 \leq p<0.10)$ Test $\times$ Group interaction, differences between pre- and post-testing values were analyzed for each group separately using paired $t$ tests. Further, effect sizes were calculated by converting partial eta-squared to Cohen's $d$. In accordance to Cohen [19], $0 \leq d \leq 0.49$ represent small effects, $0.50 \leq d \leq$ 0.79 represent moderate effects, and $d \geq 0.80$ represent large effects. All statistical analyses were performed using Statistical Package for Social Sciences version 24.0. 


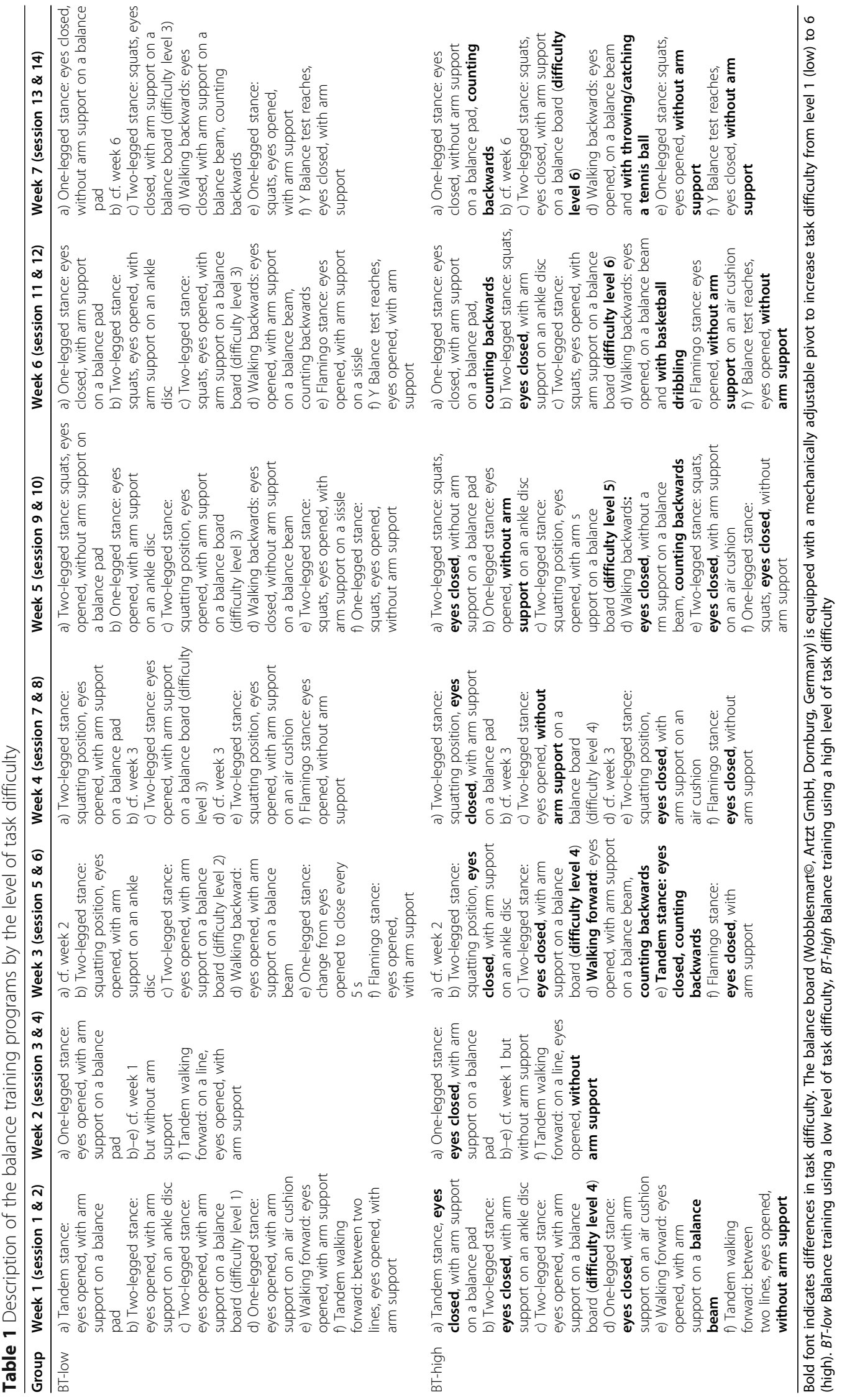




\section{Results}

Table 2 displays statistics for all analyzed variables. In general, there were no statistically significant differences in pre-test values between the two intervention groups. Further, the attendance rates during training sessions amounted to 93.5 and $93.0 \%$ in the BT-high and BT-low group, respectively.

\section{Static balance performance}

Irrespective of stance condition, the analyses revealed statistically significant main effects of Test $\left(13.391 \leq F_{1}\right.$, ${ }_{36} \leq 37.044, p \leq 0.001,1.22 \leq d \leq 2.03$ ) (Table 2). Further, a tendency toward a significant Test $\times$ Group interaction was found for standing with eyes opened on firm surface $\left(F_{1,74}=3.298, p=0.078, d=0.61\right)$. Post-hoc analyses revealed significant improvements from pre- to post-test in the BT-low $(\Delta+31 \%, p=0.003, d=0.67)$ but not in the BT-high $(\Delta+9 \%, p=0.138, d=0.30)$ group (Fig. 3$)$. In addition, a significant main effect of Group $\left(F_{1,36}=\right.$ 3.298, $p=0.037, d=0.72$ ) was observed for standing with eyes closed on firm surface. Furthermore, the number of participants that reached the maximal stance duration of $60 \mathrm{~s}$ increased from pre- to post-test in the BT-low (EOFI: from 10 to 16; EC-FI: from 0 to 2; EO-FO: from 3 to 12) and in the BT-high (EO-FI: from 13 to 15; EC-FI: from 2 to 7; EO-FO: from 5 to 11).

\section{Dynamic balance performance}

In terms of gait velocity, the analysis failed to detect significant main effects of Test and Group or a Test $\times$ Group interaction (Table 2).

\section{Proactive balance performance}

For the YBT, the analyses indicated statistically significant main effects of Test for all reach directions $\left(14.219 \leq F_{1}, \quad 36 \leq 34.767, p \leq 0.001,1.26 \leq d \leq 1.96\right)$ and the CS $\left(F_{1,36}=41.342, p<0.001, d=2.15\right)$ (Table 2). Further, a tendency toward a significant Test $\times$ Group interaction was detected for the AT reach direction $\left(F_{1}\right.$, $74=3.388, p=0.074, d=0.61)$. Post-hoc analyses yielded significant enhancements over the course of training in the BT-high $(\Delta+9 \%, p<0.001, d=0.60)$ and in the BTlow $(\Delta+5 \%, p=0.005, d=0.36)$ group (Fig. 4a). The main effects of Group were not significant.

With regard to FRT, the analysis detected a significant main effect of Test $\left(F_{1,36}=21.504, p<0.001, d=1.57\right)$ as well as a significant Test $\times$ Group interaction $\left(F_{1,74}=\right.$ 6.663, $p=0.014, d=0.87$ ) (Table 2). Post-hoc analysis found that the participants in the BT-high group significantly increased their reach distance over the training period $(\Delta+8 \%, p<0.001, d=0.35)$ while the participants in the BT-low group showed no significant changes $(\Delta+$ $2 \%, p=0.161, d=0.19$ ) (Fig. 4b). The main effect of Group was not significant.

Concerning the TUG, the analysis showed a tendency toward a significant main effect of Test $\left(F_{1,36}=2.894\right.$, $p=0.098, d=0.57$ ) (Table 2). The main effect of Group and the interaction effect of Test $\times$ Group were not significant.

\section{Discussion}

We investigated the effects of BT using a low versus a high level of task difficulty on proxies of balance

Table 2 Effects of balance training using a high versus low level of task difficulty on measures of balance in youth

\begin{tabular}{|c|c|c|c|c|c|c|c|c|c|}
\hline \multirow[t]{2}{*}{ Variables } & \multicolumn{3}{|c|}{ BT-low $(n=19)$} & \multicolumn{3}{|c|}{ BT-high $(n=19)$} & \multicolumn{3}{|c|}{$p$-value (Cohen's $d$ ) } \\
\hline & Pre & Post & $\Delta \%^{\mathrm{a}}$ & Pre & Post & $\Delta \%^{\mathrm{a}}$ & Test & Test x Group & Group \\
\hline \multicolumn{10}{|l|}{ Static balance } \\
\hline OLS time; EO, FI [s] & $44.8 \pm 19.8$ & $58.7 \pm 10.3$ & +31 & $50.4 \pm 16.1$ & $54.9 \pm 13.9$ & +9 & $.001(1.22)$ & $.078(0.61)$ & $.798(0.09)$ \\
\hline OLS time; EC, FI [s] & $13.4 \pm 11.7$ & $30.0 \pm 17.8$ & +124 & $23.7 \pm 19.2$ & $40.8 \pm 20.8$ & +72 & $<.001(1.83)$ & $.935(0.04)$ & $.037(0.72)$ \\
\hline OLS time; EO, FO [s] & $23.7 \pm 18.5$ & $49.6 \pm 17.5$ & +109 & $31.1 \pm 21.6$ & $49.4 \pm 17.4$ & +59 & $<.001(2.03)$ & $.301(0.35)$ & $.466(0.25)$ \\
\hline \multicolumn{10}{|l|}{ Dynamic balance } \\
\hline Gait velocity $[\mathrm{m} / \mathrm{s}]$ & $1.63 \pm 0.26$ & $1.66 \pm 0.22$ & +2 & $1.71 \pm 0.28$ & $1.69 \pm 0.20$ & -1 & $.909(0.05)$ & $.387(0.29)$ & $.461(0.25)$ \\
\hline \multicolumn{10}{|l|}{ Proactive balance } \\
\hline AT [\% LL] & $69.8 \pm 9.2$ & $73.2 \pm 9.7$ & +5 & $71.6 \pm 10.4$ & $78.1 \pm 11.1$ & +9 & $<.001(1.96)$ & $.074(0.61)$ & $.301(0.35)$ \\
\hline PM [\% LL] & $110.3 \pm 9.6$ & $112.5 \pm 7.7$ & +2 & $109.6 \pm 13.9$ & $114.9 \pm 10.8$ & +5 & $.001(1.26)$ & $.125(0.52)$ & $.802(0.09)$ \\
\hline$P L[\%$ LL] & $104.4 \pm 11.1$ & $109.1 \pm 7.4$ & +5 & $107.9 \pm 12.8$ & $113.1 \pm 10.6$ & +5 & $<.001(1.51)$ & $.829(0.06)$ & $.253(0.39)$ \\
\hline CS $[\% \mathrm{LL}]$ & $94.9 \pm 8.7$ & $98.3 \pm 7.0$ & +4 & $96.4 \pm 11.1$ & $102.1 \pm 9.2$ & +6 & $<.001(2.15)$ & $.110(0.54)$ & $.356(0.31)$ \\
\hline $\mathrm{FRT}[\mathrm{cm}]$ & $42.7 \pm 7.2$ & $43.6 \pm 6.4$ & +2 & $42.4 \pm 9.6$ & $45.7 \pm 9.2$ & +8 & $<.001(1.57)$ & $.014(0.87)$ & $.742(0.11)$ \\
\hline TUG [s] & $5.2 \pm 0.4$ & $5.0 \pm 0.5$ & +4 & $5.3 \pm 0.7$ & $5.2 \pm 0.5$ & +2 & $.098(0.57)$ & $.701(0.13)$ & $.521(0.22)$ \\
\hline
\end{tabular}

Values are mean values \pm standard deviations. Figures in brackets are effect sizes (Cohen's $d$ ) with $0 \leq d \leq 0.49$ indicating small, $0.50 \leq d \leq 0.79$ medium, and $d \geq$ 0.80 large effects. ${ }^{a} \mathrm{~A}$ positive/negative percentage value indicates a performance improvement/decrement. AT Anterior, $B T$-low Balance training using a low level of task difficulty, BT-high Balance training using a high level of task difficulty, CS Composite score, EC Eyes closed, EO Eyes opened, FI Firm ground, FO Foam ground, FRT Functional Reach Test, LL Leg length, OLS One-legged stance, PL Posterolateral, PM Posteromedial, TUG Timed-Up-and-Go Test 


\section{Test $x$ Group Interaction in favour of \\ BT-low $(p=0.003, d=0.67)$}

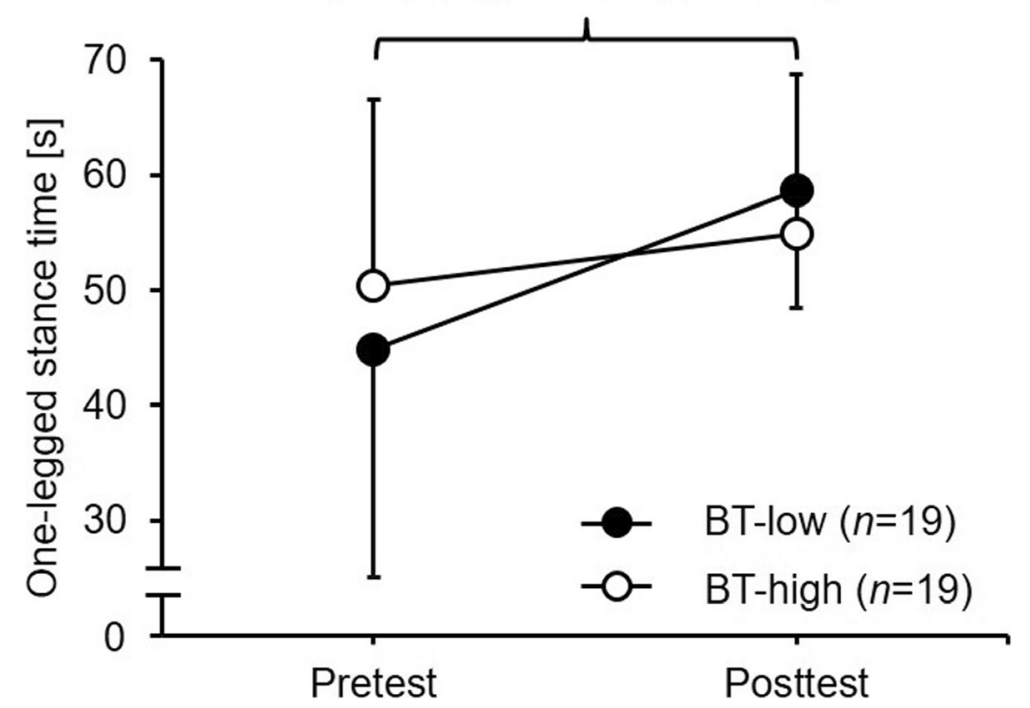

Fig. 3 Performance changes (mean \pm standard deviation) during the intervention period in static balance (i.e., one-legged stance time for the eyes opened, firm ground condition) for the BT-low compared to the BT-high group. BT-low = balance training using a low level of task difficulty; BT-high = balance training using a high level of task difficulty

performance in healthy youth. The main findings of this study can be summarized as follows: (1) in both groups, all but one (i.e., 10-m gait velocity) measure of balance performance were significantly enhanced after the training period; (2) performance improvements in one parameter of static balance (i.e., OLS with eyes opened on firm ground) were small-sized for the BT-high and medium-sized for the BT-low group; (3) performance enhancements in some parameters of proactive balance (i.e., AT reach distance and FRT distance) were small- to medium-sized for the BT-high and small-sized for the BT-low group.

\section{Effects of balance training on measures of balance performance}

In accordance with our hypothesis, we found that both exercise conditions resulted in enhanced balance performance. This finding corresponds with those from earlier studies investigating the impact of $\mathrm{BT}$ on measures of balance performance. For example, Granacher

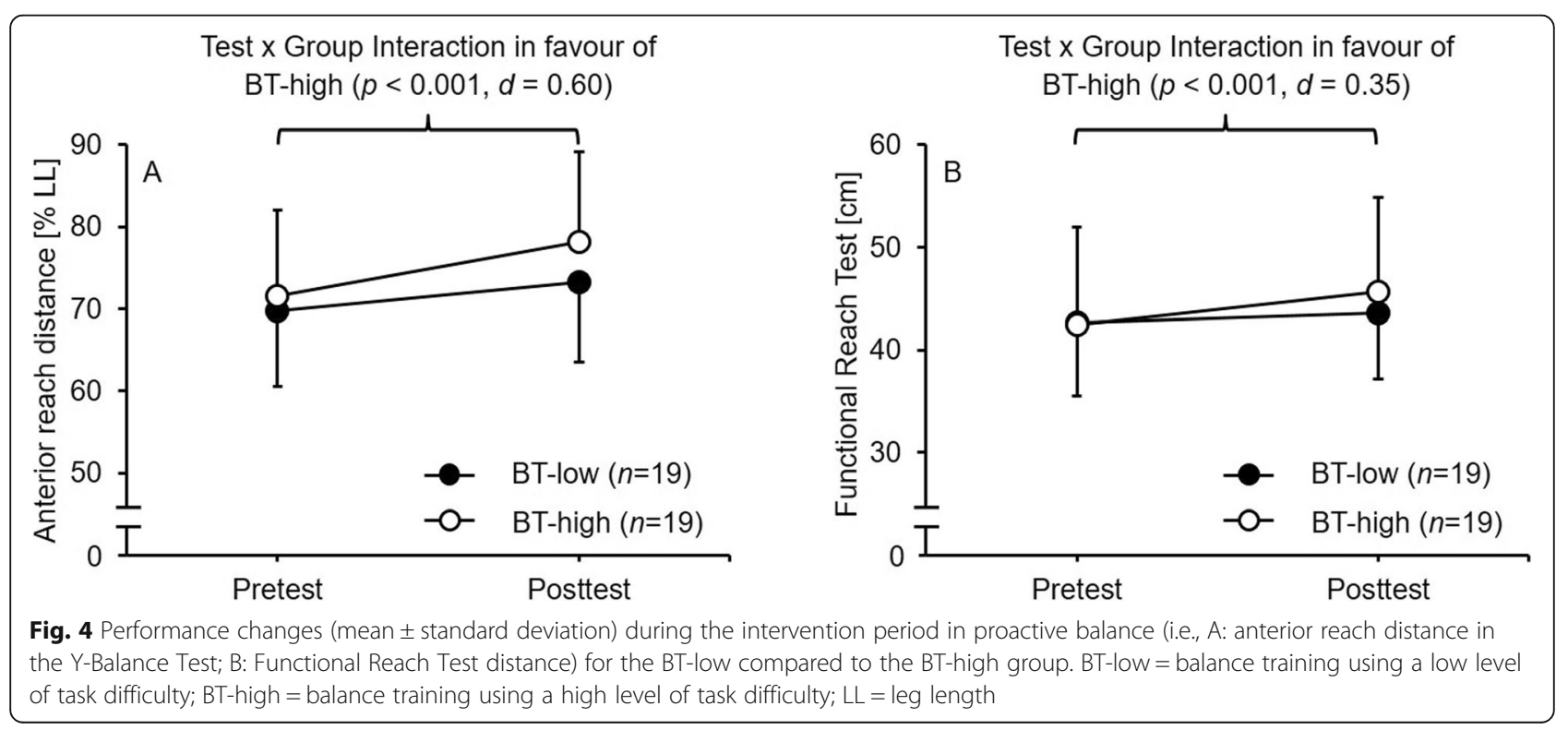


et al. [1] assigned high-school students to an intervention (BT) or an active control (P.E.) group. After 4 weeks of treatment (3 sessions per week), both groups improved their balance performance (i.e., reduced postural sway during one-legged stance) but the reductions were more pronounced in the BT compared to the P.E. group. Further, Heleno et al. [2] investigated the effect of 5 weeks (3 times per week) of BT additional to regular soccer training on proxies of static (30-s one-legged stance) and dynamic (modified Star Excursion Balance Test) balance in adolescent soccer players (mean age: 15 years). In comparison to the active control group (i.e., soccer training only), the BT plus soccer training group exhibited greater enhancements of their balance performance (i.e., less postural sway and larger reach distance) than the soccer training only group. Lastly, Pau et al. [3] examined young volleyball players (mean age: $13.0 \pm 0.2$ years) that conducted 6 weeks ( 3 times per week) of BT in addition to regular volleyball training or regular volleyball training only. They found larger improvements in static balance performance (smaller postural sway areas during 20-s two-legged and 10-s onelegged stance) for the BT plus volleyball training group compared to the volleyball training only group. The results of the present study and those of the current literature [1-3] suggest that BT is a suitable exercise regimen to improve several proxies of balance performance in healthy youth.

\section{Effects of balance training difficulty on measures of balance performance}

Partly in line with our hypothesis, we detected larger improvements in balance performance (i.e., AT reach distance and FRT distance) for the group that used a high compared to a low level of balance task difficulty during BT. There is empirical evidence [9-13] that balance performance can be challenged by the level of balance task difficulty. In this regard, Barbado Murillo et al. [11] assessed the effect of increasing difficulty in a standing balance task in young adults. As a result, the amplitude of postural sway significantly increased as the level of task difficulty changed from standing on a static surface over standing on a surface with medium followed by high instability. Further, Muehlbauer et al. [9] investigated young adults that performed the one-legged stance under various sensory conditions with an increased level of task difficulty. They found that altering the stance conditions from eyes opened on firm ground over eyes opened on foam ground to eyes closed on firm ground resulted in significant increases in postural sway. In addition, Donath and colleagues [12] examined young and older adults while they performed five standing tasks with increasing postural demand. Irrespective of the investigated age cohort, they reported significant increases in postural sway for the 30-s two-legged stance as the visual input (from eyes opened to closed) and the standing surface (from firm to foam) were changed. Lastly, Gebel et al. [13] studied the impact of graded task difficulty using a balance board with an adjustable pivot point to reduce the base of support diameter from $14 \mathrm{~cm}$ (level 1) to $4 \mathrm{~cm}$ (level 6) in adolescents. As the difficulty level changed from 1 to 6 , the postural sway significantly increased.

Besides these findings from cross-sectional studies, there is additional evidence from one intervention study [20]. More precisely, Blasco et al. [20] determined the effect of BT with different stability conditions on balance performance in young adults. Participants were assigned to $\mathrm{BT}$ groups that trained on stable ground (corresponds to BT-low) or on unstable surfaces (corresponds to BThigh). Following 3 weeks of treatment (3 sessions per week), both groups improved their balance performance (i.e., YBT and FRT reach distance, one-legged stance time). But contrary to the present study, the improvements were not statistically different between groups. This discrepancy may be explained by the longer training period in the present study (i.e., 7 weeks) as compared to the study of Blasco and colleagues (i.e., 3 weeks) as it is assumed that longer training periods evoke larger adaptations.

What are likely explanations for our observation that participants in the BT-high group achieved partly larger improvements than those in the BT-low group? In addition to assessing behavioral measures (postural sway), the aforementioned cross-sectional studies also analyzed neuromuscular measures (muscle activity). All studies reported not only significant increases in variables of postural sway but also in parameters of muscle activation as a result of increased task difficulty. Enhanced muscular activity is achieved by recruiting additional motor units, increasing the frequency and/or improved synchronization of motor units [21]. Thus, regularly performing balance tasks of high as compared to low difficulty allows larger adaptive processes, which explains the larger improvements we observed in the BT-high compared to the BT-low group. Furthermore, BT-high may elicit larger changes in sensory integration and postural control strategy compared to BT-low. In this regard, van Dieen et al. [22] assessed balance performance during single leg stance in individuals learning to balance on an unstable device while also manipulating vestibular (i.e., galvanic vestibular stimulation) and visual (i.e., moving visual field) input. Participants initially increased proprioceptive input, but with practice upweighted visual and vestibular information indicating changes in the use of sensory information. Additionally, following 6 weeks of balance training on an unstable surface (i.e., slackline) Giboin et al. [23] reported 
decreased H-reflex amplitudes during stepping on a slackline indicating a more anticipatory/feedforward organization of postural control. Another explanation relates to the central nervous plane [24-27]. For example, Gebel et al. [24] used electroencephalographic analyses to examine the impact of increasing balance task difficulty on cortical activity in adolescents. They observed significant increases in the theta frequency band power and decreases in the alpha-2 frequency band power with increasing levels of task difficulty. The authors interpreted their finding as indicating enhanced attentional processes and/or error monitoring as well as increased sensory information processing due to increasing postural demands. This would also imply larger adaptive capacities, which would also explain the superior performance of the BT-high compared to the BT-low group.

\section{Conclusions}

In the present study, we investigated the effects of BT conducted with a low versus a high level of balance task difficulty in adolescents. For both training regimens, we detected significant improvements in measures of static and proactive but not dynamic balance performance. Additionally, the enhancements in static balance were medium-sized for the BT-low and small-sized for the BT-high group. Further, the BT-low group showed small-sized and the BT-high group yielded small- to medium-sized improvements in proactive balance. Thus, it seems that BT-high is more effective for increasing measures of proactive balance, whereas BT-low is more effective for improving proxies of static balance.

\section{Abbreviations \\ ANOVA: Analysis of variance; AT: Anterior; BMI: Body mass index; BT: Balance training; BT-high: Balance training using a high level of task difficulty; BT- low: Balance training using a low level of task difficulty; CS: Composite score; EC: Eyes closed; EO: Eyes opened; Fl: Firm ground; FO: Foam ground; FRT: Functional Reach Test; LL: Leg length; OLS: One-legged stance; PL: Posterolateral; PM: Posteromedial; TUG: Timed-Up-and-Go Test}

\section{Acknowledgements}

Not applicable.

\section{Authors' contributions}

TM, FT, and LW designed the research question. FT and LW planned and supervised the interventions. FT and LW conducted the testings and data collections. TM and SS analyzed the data. TM and SS wrote the main parts of the manuscript. All the authors contributed to critical review of draft manuscripts and approved the final manuscript.

\section{Funding}

We acknowledge support by the Open Access Publication Fund of the University of Duisburg-Essen. The funding body is independent of the design of the study and collection, analysis, and interpretation of data and in writing the manuscript. Open Access funding enabled and organized by Projekt DEAL.

\section{Availability of data and materials}

The data generated and analyzed during the present study are not publicly available due to ethical restrictions but are available from the corresponding author upon reasonable request.

\section{Ethics approval and consent to participate}

Ethical permission was given by the Human Ethics Committee at the University of Duisburg-Essen, Faculty of Educational Sciences. Participants' assent and parents' written informed consent were obtained prior to the start of the study.

\section{Consent for publication}

Not applicable.

\section{Competing interests}

The authors declare that they have no competing interests. TM is an Associate Editor of the BMC Sports Science, Medicine, and Rehabilitation journal.

Received: 27 May 2020 Accepted: 4 November 2020

Published online: 23 November 2020

\section{References}

1. Granacher U, Gollhofer A, Kriemler S. Effects of balance training on postural sway, leg extensor strength, and jumping height in adolescents. Res $Q$ Exerc Sport. 2010;81(3):245-51.

2. Heleno LR, da Silva RA, Shigaki L, Araujo CG, Coelho Candido CR, Okazaki $\mathrm{VH}$, et al. Five-week sensory motor training program improves functional performance and postural control in young male soccer players - a blind randomized clinical trial. Phys Ther Sport. 2016;22:74-80.

3. Pau M, Loi A, Pezzotta MC. Does sensorimotor training improve the static balance of young volleyball players? Sports Biomech. 2012;11(1):97-107.

4. Schedler S, Brock K, Fleischhauer F, Kiss R, Muehlbauer T. Effects of balance training on balance performance in youth: are there age differences? Res $Q$ Exerc Sport. 2020:91(3):405-14

5. Gebel A, Lesinski M, Behm DG, Granacher U. Effects and dose-response relationship of balance training on balance performance in youth: a systematic review and meta-analysis. Sports Med. 2018;48(9):2067-89.

6. Gebel A, Prieske O, Behm DG, Granacher U. Effects of balance training on physical fitness in youth and young athletes: a narrative review. Strength Condition J. 2020:42(6):35-44.

7. Granacher U, Muehlbauer T, Gollhofer A, Kressig RW, Zahner L. An intergenerational approach in the promotion of balance and strength for fall prevention - a mini-review. Gerontology. 2011;57(4):304-15.

8. Granacher U, Muehlbauer T, Taube W, Gollhofer A, Gruber M. Sensorimotor training. In: Cardinale M, Newton R, Nosaka K, editors. Strength and conditioning: biological principles and practical applications. Chichester: Wiley: 2011. p. 399-409.

9. Muehlbauer T, Mettler C, Roth R, Granacher U. One-leg standing performance and muscle activity: are there limb differences. J Appl Biomech. 2014;30(3):407-14.

10. Muehlbauer T, Roth R, Bopp M, Granacher U. An exercise sequence for progression in balance training. J Strength Cond Res. 2012;26(2):568-74.

11. Barbado Murillo D, Sabido Solana R, Vera-Garcia FJ, Gusi Fuertes N, Moreno FJ. Effect of increasing difficulty in standing balance tasks with visual feedback on postural sway and EMG: complexity and performance. Hum Mov Sci. 2012;31(5):1224-37.

12. Donath L, Kurz E, Roth R, Zahner L, Faude O. Leg and trunk muscle coordination and postural sway during increasingly difficult standing balance tasks in young and older adults. Maturitas. 2016;91:60-8.

13. Gebel A, Luder B, Granacher U. Effects of increasing balance task difficulty on postural sway and muscle activity in healthy adolescents. Front Physiol. 2019:10:1135.

14. Faul F, Erdfelder E, Lang AG, Buchner A. G* power 3: a flexible statistical power analysis program for the social, behavioral, and biomedical sciences. Behav Res Methods. 2007;39(2):175-91.

15. Schulz KF, Altman DG, Moher D, Group C. CONSORT 2010 statement: updated guidelines for reporting parallel group randomized trials. Obstet Gynecol. 2010;115(5):1063-70.

16. Plisky PJ, Gorman PP, Butler RJ, Kiesel KB, Underwood FB, Elkins B. The reliability of an instrumented device for measuring components of the star excursion balance test. N Am J Sports Phys Ther. 2009:4(2):92-9.

17. Donahoe B, Turner $\mathrm{D}$, Worrell $\mathrm{T}$. The use of functional reach as a measurement of balance in boys and girls without disabilities ages 5 to 15 years. Pediatr Phys Ther. 1994;6(4):189-93. 
18. Williams EN, Carroll SG, Reddihough DS, Phillips BA, Galea MP. Investigation of the timed 'up \& go' test in children. Dev Med Child Neurol. 2005;47(8): 518-24.

19. Cohen J. Statistical power for the behavioral sciences. Hillsdale: Erlbaum; 1988.

20. Blasco JM, Tolsada C, Beltran M, Momparler AM, Sanchiz-Benavente R, Hernandez-Guillen D. Instability training, assessing the impact of level of difficulty on balance: a randomized clinical trial. Gait Posture. 2019;70:116-21.

21. Basmajian JV, de Luca CJ. Muscle alive: their functions revealed by electromyography. Baltimore: Williams \& Wilkins; 1985.

22. van Dieen $\mathrm{JH}$, van Leeuwen $\mathrm{M}$, Faber GS. Learning to balance on one leg: motor strategy and sensory weighting. J Neurophysiol. 2015;114(5):2967-82.

23. Giboin LS, Loewe K, Hassa T, Kramer A, Dettmers C, Spiteri S, et al. Cortical, subcortical and spinal neural correlates of slackline training-induced balance performance improvements. Neuroimage. 2019;202:116061.

24. Gebel A, Lehmann T, Granacher U. Balance task difficulty affects postural sway and cortical activity in healthy adolescents. Exp Brain Res. 2020;238: 1323-33.

25. Edwards AE, Guven O, Furman MD, Arshad Q, Bronstein AM. Electroencephalographic correlates of continuous postural tasks of increasing difficulty. Neuroscience. 2018;395:35-48.

26. Hulsdunker T, Mierau A, Neeb C, Kleinoder H, Struder HK. Cortical processes associated with continuous balance control as revealed by EEG spectral power. Neurosci Lett. 2015;592:1-5.

27. Hulsdunker T, Mierau A, Struder HK. Higher balance task demands are associated with an increase in individual alpha peak frequency. Front Hum Neurosci. 2015;9:695.

\section{Publisher's Note}

Springer Nature remains neutral with regard to jurisdictional claims in published maps and institutional affiliations.

Ready to submit your research? Choose BMC and benefit from:

- fast, convenient online submission

- thorough peer review by experienced researchers in your field

- rapid publication on acceptance

- support for research data, including large and complex data types

- gold Open Access which fosters wider collaboration and increased citations

- maximum visibility for your research: over $100 \mathrm{M}$ website views per year

At BMC, research is always in progress.

Learn more biomedcentral.com/submissions 